\author{
Scientific Electronic Archives \\ Issue ID: Sci. Elec. Arch. Vol. 15 (3) \\ March 2022 \\ DOI: http://dx.doi.org/10.36560/15320221515 \\ Article link: https://sea.ufr.edu.br/SEA/article/view/1515
}

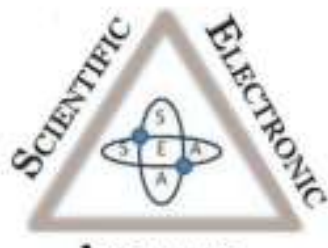

Archives

ISSN 2316-9281

\title{
Germinação de sementes de Mabea fistulifera em diferentes substratos e temperaturas
}

\author{
Germination of Mabea fistulifera seeds in different substrates and \\ temperatures
}

Corresponding author

Sérgio Roberto Garcia dos Santos

Instituto de Pesquisas Ambientais sergiorgsantos@yahoo.com.br

Renata Salaro Stecca e França de Oliveira Instituto de Pesquisas Ambientais

Sebastiania Dutra de Souza Revoredo Silva

Instituto de Pesquisas Ambientais

\begin{abstract}
Resumo. Mabea fistulifera é uma espécie florestal nativa conhecida como mamoninha-do-cerrado, cuja distribuição ocorre no sudeste do país, principalmente em áreas de transição para o Cerrado. O objetivo deste estudo foi avaliar o desempenho de diferentes substratos e temperaturas na germinação de sementes desta espécie em laboratório. Foram testados três substratos: areia, papel mata borrão e vermiculita, com as sementes instaladas entre e sobre estes. As temperaturas estudadas foram $25^{\circ} \mathrm{C}, 30^{\circ} \mathrm{C}, 20-30^{\circ} \mathrm{C}$ e $20-35^{\circ} \mathrm{C}$. Os parâmetros analisados foram a germinação e o índice de velocidade de germinação (IVG). O experimento utilizou o delineamento inteiramente casualisado, em um fatorial de $4 \times 6$ (temperaturas $x$ substratos). Com base nos resultados tem-se que os valores médios de germinação obtidos para $20-30^{\circ} \mathrm{C}$ e $20-35^{\circ} \mathrm{C}$ foram superiores aos de $30^{\circ} \mathrm{C}$. E para o IVG observou-se que: 1 - as temperaturas alternadas foram superiores estatisticamente às temperaturas constantes e 2- na média de resultados o melhor substrato é a areia (entre e sobre semeadura). Recomenda-se utilizar temperaturas alternadas $\left(20-30^{\circ} \mathrm{C} \mathrm{e} 20-35^{\circ} \mathrm{C}\right)$ e os substratos entre areia, entre papel e sobre areia.
\end{abstract}

Palavras-chave: análise de sementes; qualidade fisiológica; espécie florestal; nativa

\begin{abstract}
Mabea fistulifera is a native forest species known as mamoninha-do-cerrado, whose distribution occurs in the southeast of the country, mainly in areas of transition to the Cerrado. The aim of this study was to evaluate the performance of different substrates and temperatures on seed germination of this species in the laboratory. Three substrates were tested: sand, blotting paper and vermiculite, with the seeds installed between and on top of them. The temperatures studied were $25^{\circ} \mathrm{C}, 30^{\circ} \mathrm{C}, 20-30^{\circ} \mathrm{C}, 20-35^{\circ} \mathrm{C}$. The parameters analyzed were germination and germination speed index (IVG). The experiment used a completely randomized design, in a 4x6 factorial (temperature $x$ substrates). Based on the results, the mean germination values obtained for $20-30^{\circ} \mathrm{C}$ and $20-35^{\circ} \mathrm{C}$ were higher than those obtained at $30^{\circ} \mathrm{C}$. And for the IVG it was observed that: 1 - the alternating temperatures were statistically higher than the constant temperatures and 2- in the average of results, the best substrate is sand (between and over sowing). It is recommended to use alternating temperatures $\left(20-30^{\circ} \mathrm{C}\right.$ and $\left.20-35^{\circ} \mathrm{C}\right)$ and the substrates between sand, between paper and on sand.

Keywords: seed analysis; physiological quality; forest species; native
\end{abstract}

\section{Introdução}

Mabea fistulifera é uma espécie florestal nativa, conhecida como mamoniha-do-cerrado, cuja distribuição ocorre no sudeste do país, em áreas de cerradão e floresta estacional semidecidual, principalmente em áreas de transição entre os dois tipos de vegetação. É uma 
espécie decídua, heliófita, pioneira e xerófita. Sua madeira possui baixa durabilidade.

A $M$. fistulifera apresenta vários usos: é melífera, produz óleo e sua madeira é utilizada para lenha e carvão, além da utilização em recuperação de áreas degradadas, arborização urbana e paisagismo (LORENZI, 2002; DURIGAN et al., 2004; CAMPOS FILHO \& SARTORELLI, 2015).

$O$ teste de germinação deve refletir a qualidade das sementes e não a qualidade das condições do teste. Assim, as condições do teste devem corresponder às exigências das sementes com relação a temperatura, substrato, umidade e luz (FERRAZ \& CALVI, 2010).

$O$ fator substrato tem a finalidade de proporcionar às sementes as condições adequadas à germinação e ao desenvolvimento de plântulas (FIGLIOLIA et al., 1993), pois tem influência sobre a disponibilidade de água, gases e nutrientes e age sobre a temperatura. Dentre os comumente recomendados há uma variação entre eles na composição, toxidade às sementes, associação com patógenos, aeração e capacidade de retenção de umidade. Deve ser apropriado a cada tipo de semente e o ideal é que a umidade do substrato seja mantida até o final do teste sem que haja a necessidade de reumedecimento, pois isto pode alterar o comportamento germinativo das sementes (FLORIANO, 2004; OLIVEIRA, 2012; FIGLIOLIA, 2015).

As Regras para Análise de Sementes (RAS) indicam como substratos o papel, a areia e o solo nas duas modalidades de semeadura, entre e sobre substrato (BRASIL, 2009); a vermiculita aparece na indicação do Manual de procedimentos de análise de sementes florestais (LIMA JUNIOR, 2010).

Para espécies não listadas nas RAS, a escolha do substrato deve levar em consideração os aspectos morfológicos das sementes (principalmente tamanho e formato), suas exigências em relação à água e a luz e, também, a facilidade que o substrato oferece no momento das avaliações da germinação (FERRAZ \& CALVI, 2010).

O fator temperatura influencia a germinação de forma bem expressiva tanto por agir sobre a velocidade de absorção de água, como sobre as reações bioquímicas que determinam todo 0 processo (OLIVEIRA, 2012).

Na verdade, o processo de germinação não é senão uma sequência extremamente complexa de reações bioquímicas, pelas quais substâncias de reserva armazenadas no tecido de sustentação são desdobradas, transportadas e ressintetizadas no eixo embrionário. De maneira semelhante a uma reação química, a germinação será tanto mais rápida e o processo mais eficiente, quanto maior for a temperatura, até certo limite (CARVALHO \& NAKAGAWA, 2000).

As variações de temperatura afetam a velocidade, a porcentagem e a uniformidade de germinação. Em função de sua relação com esses parâmetros, há necessidade de serem determinadas temperaturas em que a eficiência do processo é total, bem como os extremos (máximo e mínimo) tolerados pelas sementes. Há também espécies que exigem temperaturas alternadas, este comportamento está relacionado a falta de domesticação destas e associado à aquelas que apresentam dormência e as razões que determinam os efeitos da alternância de temperatura não são totalmente conhecidas, mas supõe-se que esta variação térmica cria uma alteração no balanço entre promotores e inibidores da germinação (MARCOS FILHO, 2005).

Com base na importância destes dois fatores, o objetivo deste estudo foi avaliar o desempenho de diferentes substratos e temperaturas na germinação em laboratório de sementes de Mabea fistulifera.

\section{Métodos}

Foram testados três substratos: areia, papel mata borrão e vermiculita, com as sementes (colhidas em Assis/SP) semeadas entre e sobre os mesmos. As temperaturas estudadas foram $25^{\circ} \mathrm{C}$, $30^{\circ} \mathrm{C}, 20-30^{\circ} \mathrm{C}$ e $20-35^{\circ} \mathrm{C}$. Cada tratamento teve quatro repetições de 25 sementes cada.

As sementes utilizadas neste estudo foram imersas em hipoclorito de sódio à $2 \%$ por 10 minutos e lavadas por três vezes com água destilada para o controle de microorganismos, bactérias e fungos (RIBEIRO et al., 2009).

Foi determinado o teor de água do lote de sementes utilizado no ensaio, pelo método de estufa a $105^{\circ} \mathrm{C} \pm 3^{\circ} \mathrm{C}$, por 24 horas (BRASIL, 2009), utilizando-se duas repetições de 25 sementes.

Os parâmetros analisados foram a germinação $e$ o İndice de Velocidade de Germinação (IVG), sendo que este índice foi calculado de acordo com a fórmula proposta por Maguire (1962). O critério adotado para considerar como germinada a semente foi o botânico, proposto por Laboriau (1983), quando há a emergência da radícula em torno de $0,5 \mathrm{~cm}$.

$\mathrm{O}$ experimento utilizou $\mathrm{o}$ delineamento inteiramente casualizado de acordo com Gomes (2009), em um fatorial de $4 \times 6$ (temperaturas $x$ substratos). As análises referentes à porcentagem de germinação foram efetuadas com os resultados transformados em arco seno. As médias foram comparadas pelo teste de Tukey (5\%).

\section{Resultados e discussão}

$O$ valor do teor de água das sementes utilizadas neste estudo foi de $8,33 \%$, adequado para sementes que estavam armazenadas e do Coeficiente de Variação (CV), foi de $12,93 \%$ para o parâmetro percentual de sementes germinadas e de $20,89 \%$ para o IVG, sendo que o CV fornece uma indicação de precisão do ensaio e foram considerados médios de acordo com tabela proposta por Pimentel-Gomes \& Garcia (2002). 
Os melhores resultados médios de germinação (Tabela 1) são para as temperaturas de $20-30^{\circ} \mathrm{C}$ e $20-35^{\circ} \mathrm{C}$ (embora equivalentes à $25^{\circ} \mathrm{C}$ ) sendo superiores ao obtido à $30^{\circ} \mathrm{C}$ e ao mesmo tempo quando se observa os resultados dos substratos tem-se que na média os dois melhores são SP e SA, embora não difiram estatisticamente de EP, sendo superiores a todos os demais.

Ao se fixar os melhores substratos e temperaturas, com base nos resultados médios de germinação, observam-se nesta Tabela 1 as seguintes informações. Para a temperatura de 20$30^{\circ} \mathrm{C}$, o substrato SP é superior aos demais (embora equivalente estatisticamente a $S A$ ) e para 20-35 ${ }^{\circ} \mathrm{C}, \mathrm{EP}, \mathrm{EA}$ e SP, são os melhores substratos, sendo equivalentes entre si, e para esta temperatura, destaca-se EP, sendo superior aos demais substratos. Para o substrato SP, o maior valor de temperatura é $20-30^{\circ} \mathrm{C}$, embora não difira estatisticamente de $20-35^{\circ} \mathrm{C}$, sendo esta superior às constantes de $25^{\circ} \mathrm{C}$ e $30^{\circ} \mathrm{C}$. E para o substrato $\mathrm{SA}$, as temperaturas $25^{\circ} \mathrm{C}, 30^{\circ} \mathrm{C}$ e $20-30^{\circ} \mathrm{C}$ são estatisticamente equivalentes, sendo que esta última é superior a alternada de $20-35^{\circ} \mathrm{C}$.

Quando se considera a velocidade de germinação (Tabela 2) tem-se que os melhores resultados médios são para os substratos EA e SA e para as temperaturas $20-30^{\circ} \mathrm{C}$ e $20-35^{\circ} \mathrm{C}$. Sendo que os melhores resultados dentro de EA são para as temperaturas de $25^{\circ} \mathrm{C} ; 20-30^{\circ} \mathrm{C}$ e $20-35^{\circ} \mathrm{C}$; e para SA são para $25^{\circ} \mathrm{C} ; 30^{\circ} \mathrm{C}$ e $20-30^{\circ} \mathrm{C}$. E quando se considera as melhores médias de temperaturas, tem-se que para $20-30^{\circ} \mathrm{C}$, os maiores valores são:
$S A$ e EA, embora não difiram de EP; e para 20$35^{\circ} \mathrm{C}$, os substratos EV, EA, EP e SA.

Neste estudo destacaram-se, com os melhores resultados percentuais e de velocidade de germinação, as temperaturas alternadas e os substratos areia e papel.

A interação significativa entre substratos e temperaturas fica clara quando ao se aumentar a amplitude da temperatura alternada (tem-se uma maior evaporação de água no substrato), neste caso os melhores tratamentos são na modalidade entre semeadura (EP e EA). Melhor esclarecendo, para a temperatura alternada com maior amplitude $\left(20-35^{\circ} \mathrm{C}\right)$, entre a mínima e a máxima temperatura, destacou-se a semeadura entre substratos, indicando uma coerência de resultados, pois a evaporação de água deve aumentar em razão do aumento de $5^{\circ} \mathrm{C}$ na temperatura máxima, mas mantendo-se a mínima. Deste modo, esta modalidade de semeadura, evita melhor a perda de água no substrato (FIGLIOLIA et al., 1993). Enquanto que na temperatura alternada, com menor amplitude $\left(20-30^{\circ} \mathrm{C}\right)$, a modalidade de semeadura sobre substrato apresentou os melhores resultados.

Vários autores também constataram a interação significativa entre temperatura e substrato. Como exemplos tem-se os estudos de Albuquerque et al. (1998) com Colubrina glandulosa; Alves et al. (2002) com Mimosa caesalpiniaefolia; Lima et al. (2007) com Bixa orellana; Oliveira et al. (2016) com Simira gardneriana e Gomes et al. (2016) para Campomanesia xanthocarpa.

Tabela 1. Valores de germinação de sementes de Mabea fistulifera obtidos em diferentes temperaturas e substratos.

\begin{tabular}{|c|c|c|c|c|c|}
\hline \multirow[b]{2}{*}{ Substrato } & \multicolumn{5}{|c|}{ Temperatura } \\
\hline & $25^{\circ} \mathrm{C}$ & $30^{\circ} \mathrm{C}$ & $20-30^{\circ} \mathrm{C}$ & $20-35^{\circ} \mathrm{C}$ & Média \\
\hline EV & $51,24 \mathrm{Aa}$ & $52,97 \mathrm{ABa}$ & $47,59 \mathrm{Ca}$ & $53,12 \mathrm{BCa}$ & $51,23 \mathrm{D}$ \\
\hline EA & $55,92 A a b$ & $44,10 \mathrm{Bb}$ & $58,49 \mathrm{BCa}$ & $57,40 \mathrm{ABCab}$ & $53,98 \mathrm{BCD}$ \\
\hline EP & $58,10 A b$ & $52,38 \mathrm{ABb}$ & $57,91 \mathrm{BCb}$ & $72,29 A a$ & $60,17 \mathrm{ABC}$ \\
\hline SV & $59,28 \mathrm{Aa}$ & $52,27 \mathrm{ABa}$ & $47,07 \mathrm{Ca}$ & $55,09 \mathrm{BCa}$ & $53,43 C D$ \\
\hline SA & 62,20 Aab & 65,57Aab & $71,42 \mathrm{ABa}$ & $49,43 \mathrm{Cb}$ & $61,40 \mathrm{AB}$ \\
\hline SP & $57,29 A b c$ & $50,78 \mathrm{ABc}$ & $74,11 \mathrm{Aa}$ & 65,07ABab & $61,81 \mathrm{~A}$ \\
\hline Média & $57,34 \mathrm{AB}$ & $52,51 \mathrm{~B}$ & $59,43 \mathrm{~A}$ & $58,73 A$ & \\
\hline \multicolumn{6}{|c|}{$\begin{array}{l}\text { F para temperatura }(\mathrm{T}): 4,45^{\star \star} ; \mathrm{F} \text { para substrato }(\mathrm{S}): 12,60^{\star *} ; \mathrm{F} \text { para interação }(\mathrm{S} \times \mathrm{T}): 3,17^{\star \star} \text {; Coeficiente de Variação } \\
(\%): 12,93\end{array}$} \\
\hline
\end{tabular}

Os bons resultados obtidos neste estudo com os substratos areia e papel, em contraposição aos resultados gerais obtidos pelo substrato vermiculita, concordam com as características da área de ocorrência da Mabea fistulifera (DURIGAN et al.,2004), ou seja, para as espécies de locais mais secos, como as florestas estacionais semideciduais e o cerrado, e com sementes pequenas (que é o caso desta espécie), recomenda-se o uso do substrato papel (PIÑARODRIGUES et al., 2004). E do mesmo modo, a areia (assim como o papel), é considerado um substrato com baixa capacidade de retenção de água (KÄMPF, 2000), melhor refletindo um 
ambiente mais seco. A vermiculita, por sua vez, não apresentou bons resultados neste estudo, quando comparada aos obtidos pelos substratos areia e papel provavelmente em razão de suas características, pois é um material com grande capacidade de retenção de umidade (FIGLIOLIA \& PIÑA-RODRIGUES, 1995; OLIVEIRA, 2012).

Os solos do bioma cerrado, segundo Coutinho (2002) são profundos porosos, permeáveis, bem drenados e por isso profundamente lixiviados. $\mathrm{Em}$ sua textura predomina, em geral, a fração areia e, por último, o silte. Eles são, portanto, predominantemente arenosos, areno-argilosos ou, eventualmente argiloarenosos. Sua capacidade de retenção de água é relativamente baixa.

Com relação às temperaturas, as alternadas $20-30^{\circ} \mathrm{C}$ e $20-35^{\circ} \mathrm{C}$, apresentaram resultados expressivos e significativos neste estudo. Estes resultados diferem da indicação de temperatura, para $M$. fistulifera, nos trabalhos de outros autores, onde prevalecem as constantes. Gomes Junior (2011) indicando a temperatura de $30^{\circ} \mathrm{C}$ e o substrato SP e Leal Filho \& Borges (1992) as temperaturas de $25^{\circ} \mathrm{C}$ e $30^{\circ} \mathrm{C}$; em ambos estudos, estes foram os melhores resultados.

$$
\text { Em outras espécies de cerrado }
$$

(MEDEIROS, 2011; DURIGAN et al., 2011; BARBOSA et al., 2015), há indicação de uso de temperaturas alternadas para a germinação de sementes, como ocorre com Rapanea umbellata $\left(20-35^{\circ} \mathrm{C}\right)$ e Syagrus romanzoffiana (20$\left.30^{\circ} \mathrm{C}\right)$, citadas nas "Instruções para análise de sementes de espécies tropicais" (BRASIL, 2013) e com SALOMÃO et al. (2003) da Rede de Sementes do Cerrado que também citaram várias espécies deste bioma (Myracrodruon urundeuva; Sterculia striata; Miconia fallax; M. ferruginosa; Eugenia dysenterica e Aegiphilla sellowiana).

O uso de temperaturas alternadas para espécies da floresta estacional semidecidual (DURIGAN et al., 2002; BARBOSA et al., 2015), também é indicado nas "Instruções para análise de sementes de espécies tropicais" (BRASIL, 2013) para Esenbeckia leiocarpa $\left(20-30^{\circ} \mathrm{C}\right.$ e $\left.25-35^{\circ} \mathrm{C}\right)$; Myracrodruon urundeuva $\left(20-30^{\circ} \mathrm{C}\right)$; Cordia trichotoma $\left(20-30^{\circ} \mathrm{C}\right)$ e Aegiphilla sellowiana (20$\left.30^{\circ} \mathrm{C}\right)$.

Com base nos resultados médios obtidos neste estudo, duas temperaturas se sobressaíram, a de $20-30^{\circ} \mathrm{C}$ e a de $20-35^{\circ} \mathrm{C}$ e os substratos areia e papel, sendo que a escolha da modalidade de semeadura irá depender da temperatura alternada utilizada. Estes dois fatores ambientais são importantes no processo de germinação, pois permitem aperfeiçoar a quantidade, velocidade e uniformidade de germinação (SILVA et al., 2014), sendo que para as espécies florestais ainda é escassa uma metodologia padronizada para a análise de sementes, sendo necessários estudos sobre as condições ecofisiológicas ótimas de germinação, dando ênfase aos efeitos de temperatura e substrato (FREIRE et al, 2011).

A espécie Mabea fistulifera ainda não tem registrado a(s) temperatura(s) e substrato(s) adequados à germinação de suas sementes, em duas publicações de interesse para sementes florestais, que são: a) Instruções para análise de sementes florestais (BRASIL, 2013) e b) Manual de Procedimentos para Análise de Sementes Florestais (LIMA JUNIOR, 2010), neste último consta somente informações sobre a temperatura.

Tabela 2. Valores do Índice de Velocidade de Germinação (IVG) de sementes de Mabea fistulifera obtidos em diferentes temperaturas e substratos.

\begin{tabular}{|c|c|c|c|c|c|}
\hline \multirow[b]{2}{*}{ Substrato } & \multicolumn{5}{|c|}{ Temperatura } \\
\hline & $25^{\circ} \mathrm{C}$ & $30^{\circ} \mathrm{C}$ & $20-30^{\circ} \mathrm{C}$ & $20-35^{\circ} \mathrm{C}$ & Média \\
\hline EV & 0,87ABa & 1,02Aa & $0,90 \mathrm{Ca}$ & $1,04 \mathrm{ABa}$ & $0,96 \mathrm{BC}$ \\
\hline EA & $1,27 \mathrm{Aa}$ & $0,87 \mathrm{ABb}$ & $1,37 \mathrm{ABa}$ & $1,30 \mathrm{Aa}$ & $1,20 \mathrm{~A}$ \\
\hline EP & $0,68 \mathrm{Bb}$ & $0,86 \mathrm{ABb}$ & 1,03ABCab & $1,27 \mathrm{Aa}$ & $0,96 \mathrm{BC}$ \\
\hline SV & 0,96ABa & $0,83 \mathrm{ABa}$ & $0,75 \mathrm{Ca}$ & 0,83Ba & $0,84 \mathrm{C}$ \\
\hline SA & 1,15Aab & 1,20Aab & $1,42 \mathrm{Aa}$ & $0,91 \mathrm{ABb}$ & $1,17 \mathrm{AB}$ \\
\hline SP & 0,60Bab & $0,55 \mathrm{Bb}$ & 0,98BCa & 0,82Bab & $0,73 \mathrm{D}$ \\
\hline Média & $0,92 \mathrm{~B}$ & $0,89 \mathrm{~B}$ & $1,07 \mathrm{~A}$ & $1,03 \mathrm{AB}$ & \\
\hline \multicolumn{6}{|c|}{$\begin{array}{l}\text { F para temperatura }(\mathrm{T}): 4,45^{\star \star} ; \mathrm{F} \text { para substrato }(\mathrm{S}): 12,60^{\star \star} ; \mathrm{F} \text { para interação }(\mathrm{S} \times \mathrm{T}): 3,17^{\star \star} \text {; Coeficiente de Variação } \\
(\%): 20,89 \\
\text { (\% Significativo pelo teste } \mathrm{F} \text { a } 1 \% \text { de probabilidade. Médias seguidas pela mesma letra maiúscula na coluna (comparam substratos } \\
\text { para cada temperatura) e minúscula na linha (comparam temperatura para cada substrato) não diferem entre si pelo Teste de Tukey a } \\
5 \% \text { de probabilidade. }\end{array}$} \\
\hline
\end{tabular}

\section{Conclusão}

Os maiores valores médios de germinação para temperatura foram $20-30^{\circ} \mathrm{C}$ e $20-35^{\circ} \mathrm{C}$ e para substrato: SP e SA
Para IVG, os maiores valores para temperatura foram $20-35^{\circ} \mathrm{C}$ e $20-35^{\circ} \mathrm{C}$ e para os substratos foram EA e SA. 
Dentro de cada parâmetro, os fatores médios de temperatura e substrato de maiores valores, foram para:

- Germinação: $\mathbf{2 0}-\mathbf{3 0}{ }^{\circ} \mathrm{C}$ - SP e SA; $20-35^{\circ} \mathrm{C}$ - EP, EA e SP. SP $-20-30^{\circ} \mathrm{C}$ e $20-35^{\circ} \mathrm{C}$; SA $-20-30^{\circ} \mathrm{C}$ é superior estatisticamente a $20-35^{\circ} \mathrm{C}$, mas equivalente a $25^{\circ} \mathrm{C}$ e $30^{\circ} \mathrm{C}$.

- IVG: 20-30 ${ }^{\circ} \mathrm{C}$ - SA, EA e EP; 20-35 ${ }^{\circ} \mathrm{C}$ - EV, EA, EP e AS. EA $-25^{\circ} \mathrm{C} ; 20-30^{\circ} \mathrm{C}$ e $20-30^{\circ} \mathrm{C}$; SA $-25^{\circ} \mathrm{C}$; $30^{\circ} \mathrm{C}$ e $20-35^{\circ} \mathrm{C}$

\section{Referências}

ALVES, E.U.; PAULA, R.C.; OLIVEIRA, A.P.; BRUNO, R.L.A.; DINIZ, A.A. Germinação de sementes de Mimosa caesalpiniifolia Benth. em diferentes substratos e temperaturas. Revista Brasileira de Sementes, v.24, n.1, p.169-178, 2002.

ALBUQUERQUE, M.C.F.; RODRIGUES, T.J.D.; MINOHARA, L.; TEBALDI, N.D.; SILVA, L.M.M. Influência da temperatura e do substrato na germinação de sementes de saguaraji (Colubrina glandulosa Perk. - Rhamnaceae). Revista Brasileira de Sementes, v. 20, n.2, p.108-111, 1998.

BARBOSA L.M.; SHIRASUNA, R.T.; LIMA, F.C.; ORTIZ, P.R.T. Lista de espécies indicadas para restauração ecológica para diversas regiões do Estado de São Paulo. In: SIMPÓSIO DE RESTAURAÇÃO ECOLÓGICA, 6, 2015, São Paulo. Anais... São Paulo: Instituto de Botânica de São Paulo, 2015. p. 303-436.

BRASIL. Ministério da Agricultura e Reforma Agrária. Regras para a Análise de Sementes. Brasília: SNDA/DNDV/CLAV, 2009. 399p.

BRASIL. Ministério da Agricultura, Pecuária e Abastecimento. Instruções para análise de sementes de espécies florestais. Ministério da Agricultura, Pecuária e Abastecimento. Secretaria de Defesa Agropecuária/Coordenação Geral de Apoio Laboratorial. Brasília, DF: Mapa/SDA/CGAL, 2013, 97p.

CAMPOS FILHO, E.M.; SARTORELLI, P.A.R. Guia de árvores com valor econômico. São Paulo: Agroicone, 2015. 139p.

CARVALHO, N.M.; NAKAGAWA, J. Sementes: ciência, tecnologia e produção. 4.ed. Jaboticabal: Funep, 2000. 588p.

COUTINHO, L. M. O bioma do cerrado. In: KLEIN, A. L. (Org.). Eugen Warming e o cerrado brasileiro: um século depois. São Paulo: Editora Unesp, Imprensa Oficial do Estado, p. 77-91, 2002.

DURIGAN, G.; SANTOS, J.D.; GANDARA, F.B. Fitossociologia de dois fragmentos de Floresta Estacional Semidecidual no Pontal do
Paranapanema, SP. Revista do Instituto Florestal. São Paulo, V.14, n.1, p. 13-26. 2002.

DURIGAN, G.; BAITELLO, J.B.; FRANCO, G.A.D.C.; SIQUEIRA, M.F. Plantas do cerrado paulista: imagens de uma paisagem ameaçada. São Paulo: Páginas \& Letras, 2004. 475p.

DURIGAN, G.; MELO, A.C.G.; MAX, J.C.M.; VILAS BÔAS, O.; CONTIERI, W.A. Manual para recuperação da vegetação de cerrado. São Paulo: $3^{\underline{a}}$ ed. SMA. 2011. 19p.

FERRAZ, I.D.K.; CALVI, G.P. Teste de germinação. In: LIMA JÚNIOR, M.J.V. (Ed.). Manual de procedimentos de análise de sementes florestais. Londrina: ABRATES, 2010. p.55-122.

FIGLIOLIA, M.B. Teste de Germinação. In: PiñaRodrigues, F.C.M; Figliolia, M.B; Silva, A. Sementes Florestais Tropicais: da ecologia à produção. Londrina: ABRATES, p. 325-343, 2015.

FIGLIOLIA, M.B.; OLIVEIRA, E.C.; PIÑARODRIGUES, F.C.M. Análise de sementes. In: AGUIAR, I.B.; PIÑA-RODRIGUES, F.C.M. \& FIGLIOLIA, M.B. (eds.). Sementes florestais tropicais. Brasília: ABRATES, 1993. p.137-174.

FIGLIOLIA, M.B.; PIÑA-RODRIGUES, F.C.M. Considerações práticas sobre 0 teste de germinação. In: SILVA, A.; PIÑA-RODRIGUES, F.C.M. \& FIGLIOLIA, M.B. (eds.). Manual técnico de sementes florestais. São Paulo: Instituto Florestal, 1995. p.45-59. (Série Registros, 14).

FLORIANO, E.P. Germinação e dormência de sementes florestais. Santa Rosa: Associação de Pesquisa, Educação e Proteção Ambiental do Noroeste do Estado do Rio Grande do Sul ANORGS, 2004. 19p. (Caderno Didático, n. 2).

FREIRE, J.M.; ROUWS, J.R.C.; TEIXEIRA, R.O. Influência do substrato no vigor de sementes de leiteira (Tabernaemontana laeta Mart.) em condições de laboratório. Boletim de Pesquisa e Desenvolvimento. Seropédica: Embrapa Agrobiologia. n.78, 2011. 20p.

GOMES, F.P. Curso de estatística experimental. Piracicaba: ESALQ, 2009, 451p.

GOMES, J.P., OLIVEIRA, L.M., FERREIRA, P.I., BATISTA, F. Substratos e temperaturas para teste de germinação em sementes de Myrtaceae. Ciência Florestal, n.26, v.4, p. 285-293. 2016.

GOMES JÚNIOR, D. Qualidade fisiológica de sementes e produção de mudas de Mabea fistulifera Mart. 2011. 82 f. Dissertação (Mestrado em Ciências Florestais) Universidade Federal do Espírito Santo, Alegre-ES. 2011. 
KÄMPF, A.N. Produção comercial de plantas ornamentais. Guaíba: Agropecuária, 2000. 254 p.

LABOURIAU, L. G. A germinação da semente. Washington: Secretaria Geral da Organização dos estados Americanos, 1983. 173p.

LEAL FILHO, N.; BORGES, E.E.L. Influência da temperatura e da luz na germinação de sementes de canudo de pito (Mabea fistulifera Mart.). Revista Brasileira de Sementes. ABRATES: Londrina, v.14, n.1, p.57-60, 1992.

LIMA, R.V.; LOPES, J.C.; COELHO, R.I. Germinação de sementes de urucu em diferentes temperaturas e substratos. Ciência e Agrotecnologia, v.31, n.4, p.1219-1224, 2007.

LIMA JUNIOR, M.J.V. (Ed.) Manual de Procedimentos para Análise de Sementes Florestais. UFAM: Manaus, 2010. 146p.

LORENZI, H. Árvores Brasileiras: Manual de Identificação e Cultivo de Plantas Arbóreas Nativas do Brasil. Piracicaba/SP, 2002. 384p.

MAGUIRE, J.D. Speed of germination-aid in selection and evaluation for seedling emergence and vigor. Crop Science, Madison, v. 2, n.1, p. 176177, 1962.

MARCOS FILHO, J. Fisiologia de sementes de plantas cultivadas. Piracicaba: FEALQ, 2005. 495p.

MEDEIROS, J.D. Guia de campo: vegetação do cerrado - 500 espécies. Brasilia: MMA/SBF. 2011. 532p. (Série Biodiversidade, 43)

OLIVEIRA, F.N.; FRANÇA, F.D.; TORRES, S.B.; NOGUEIRA, N.W.; FREITAS, R.M.O. Temperaturas e substratos na germinação de sementes de pereiro-vermelho (Simira gardneriana M.R. Barbosa \& Peixoto). Revista Ciência Agronômica, v. 47, n. 4, p. 658-666, 2016.

OLIVEIRA, O.S. Tecnologia de sementes florestais: espécies nativas. Curitiba: UFPR, 2012, 403p.

PIMENTEL-GOMES, F.; GARCIA, C.R. Estatística aplicada a experimentos agronômicos e florestais: exposição com exemplos e orientações para uso de aplicativos. Piracicaba: FEALQ. 2002. 309p

PIÑA-RODRIGUES, F.C.M.; FIGLIOLIA, M.B.; PEIXOTO, M.C. Teste de qualidade. In: Ferreira, A.G.; Borghetti, F. (Org.) Germinação: do básico ao aplicado. Porto Alegre: Artmed, 2004. p.283- 297.

RIBEIRO, M.F., CUCHIARA, C.C.; BORGES, C.S.; OLIVEIRA-NAPOLEÃO, I.T.; MOURA, A.B.; MORAES, D.M. Influência do hipoclorito de sódio na germinação e vigor de sementes de arroz (Oriza sativa L.). Resumos... XVIII CONGRESSO DE INICIAÇÃO CIENTÍFICA - CIC UFPel, Pelotas, 2009.

SALOMÃO, A.N., SOUSA-SILVA, J.C., DAVIDE, A.C., GONZÁLES, S., TORRES, R.A.A., WETZEL, M.M.V.S., FIRETTI, F. \& CALDAS, L.S. Germinação de Sementes e Produção de Mudas e Plantas do Cerrado (A. N. Salomão et al., ed.). Rede de Sementes do Cerrado, Brasília, 2003. 96p.

SILVA, L.M.M.; ALMEIDA, F.A.C.; QUEIROGA, V.C. Germinação de sementes. in: Sementes: estudos tecnológicos. Aracaju: IFS, 2014. p.85-97. 